\title{
The Trend in the Sales of Menopausal Hormone and Other Osteoporosis Medications in South Korea from 2016 to 2019
}

\author{
Eun Sil Lee', Sogene Kwon'1, Hyoung Moo Park ${ }^{2}$ \\ 'Department of Obstetrics and Gynecology, Soonchunhyang University Seoul Hospital, Soonchunhyang University College of Medicine, \\ Seoul; \\ ${ }^{2}$ Department of Obstetrics and Gynecology, Menopause Clinic Grace Women's Hospital, Goyang, Korea
}

Corresponding author

Hyoung Moo Park

Department of Obstetrics and Gynecology,

Menopause Clinic Grace Women's Hospital,

1073 Junaang-ro, Ilsandong-gu, Goyang

10447, Korea

Tel: +82-31-901-4000

Fax: +82-2-709-9332

E-mail: hmpark52@hanmail.net

Received: January 6, 2021

Revised: May 18, 2021

Accepted: May 29, 2021
Background: Increasing geriatric population, osteoporosis prevalence, and interest in bone health are key contributing factors for the growth of osteoporosis medication markets. Thus, this study evaluated changes in the menopausal hormone and other osteoporosis medication markets from 2016 to 2019. Methods: This study's dataset was obtained from the International Marketing Services of IQVIA Inc. in South Korea. The sales of medications for osteoporosis treatment with menopausal hormones were evaluated for drug sales for osteoporosis treatment from 2016 to 2019. Results: The results showed that the tissue-selective estrogen complex (TSEC) sales had increased annually while the estrogen-progesterone therapy (EPT) sales had decreased. Excluding menopausal hormones, bisphosphonates were the most widely sold medications for osteoporosis treatment. Among the bisphosphonate medications, sales of ibandronate and zoledronate increased annually, while alendronate and risedronate decreased. Teriparatide also showed increasing sales. A rapid rise was noted in the sales of denosumab. Conclusions: While the sales of TSEC, injectable bisphosphonates, and denosumab have increased annually, the sales of EPT, estrogen therapy, oral bisphosphonates have not increased, as reflected in hormone therapy and osteoporosis medication market trends. This study showed the recent trends in hormone therapy and the osteoporosis medication market from 2016 to 2019 in South Korea.

Key Words: Commerce · Hormone replacement therapy · Menopause · Osteoporosis

\section{INTRODUCTION}

Korea is recently experiencing an increasing trend of osteoporosis and osteoporosis-related fracture cases.[1] According to the reimbursement records from the Korean National Health Insurance Service database in 2012, the prevalence of osteoporosis in men and women over 50 years old were $6.1 \%$ and $33.3 \%$, respectively.[2] Another study by Shin et al. [3] reported that $24.3 \%$ of women and $13.1 \%$ of men ages 40 to 79 years old in Korea have osteoporosis at any of the following sites: lumbar spine, femoral neck, or total hip.

The management of osteoporosis requires either nonpharmacologic or pharmacologic therapy. In terms of nonpharmacologic options, resistance and weightbearing exercises can increase muscle mass and bone mineral density (BMD).[4] 
Pharmacologic treatments are classified as either antiresorptive agents or anabolic agents. Antiresorptive agents, which inhibit resorption and increase bone strength, include estrogen treatment with or without progestogen, selective estrogen receptor modulators (SERMs), bisphosphonates, and denosumab, a receptor activator of nuclear factor-KB ligand (RANKL) inhibitor. On the other hand, anabolic agents work primarily by increasing bone formation rather than decreasing resorption, such as teriparatide, a parathyroid hormone (PTH), and romosozumab, a sclerostin inhibitor.[5,6]

In 2014, a menopausal hormone called tissue-selective estrogen complex (TSEC) was introduced in Korea. Then, in 2016, an osteoporosis medication called denosumab was introduced. The introduction of new osteoporosis medication is expected to change the sales of menopausal hormone and osteoporosis medications. For the reasons stated above, this study aimed to evaluate the changes in menopausal hormone and other osteoporosis medication markets from 2016 to 2019.

\section{METHODS}

Marketing and sales data of postmenopausal hormone and osteoporosis medications, between 2016 and 2019, in Korea were obtained from the International Marketing Services of IQVIA Inc. Formerly Quintiles and IMS Health, IQVIA Inc. is an American multinational company known for its collection of healthcare spanning sales, de-identified pre- scription data, medical claims, and electronic medical records. IQVIA datasets have been used by companies to select patient and physician populations for specific therapies and to measure the effectiveness of pharmaceutical marketing and sales resources. The marketing and sales data of postmenopausal hormone and osteoporosis medications were used in this study.

The use of medication for osteoporosis treatment with postmenopausal hormone was evaluated based on osteoporosis treatment drug sales using the drug sales audit from IQVIA to analyze specific drugs' sales. These drugs were categorized based on drug types, such as postmenopausal hormones, bisphosphonates, SERMs, PTH (teriparatide), and RANKL inhibitors (denosumab). The postmenopausal hormones include estrogen, estrogen combined with progestogen, tibolone, and TSEC. Bisphosphonates consist of alendronate, oral and injectable ibandronate, risedronate, and zoledronate. Lastly, SERMs include raloxifene and bazedoxifene. Then, the total annual sales (in USD) and units sold were analyzed. This study was granted exemption by the Institutional Review Board (IRB) of Soonchunhyang University Seoul Hospital (SCH IRB no. 2021-02-017).

\section{RESULTS}

\section{Postmenopausal hormone treatment}

Postmenopausal hormones include estrogen-progestogen therapy (EPT), tibolone, TSEC, and estrogen therapy (ET). Based on Figure 1, the sales of TSEC had increased from
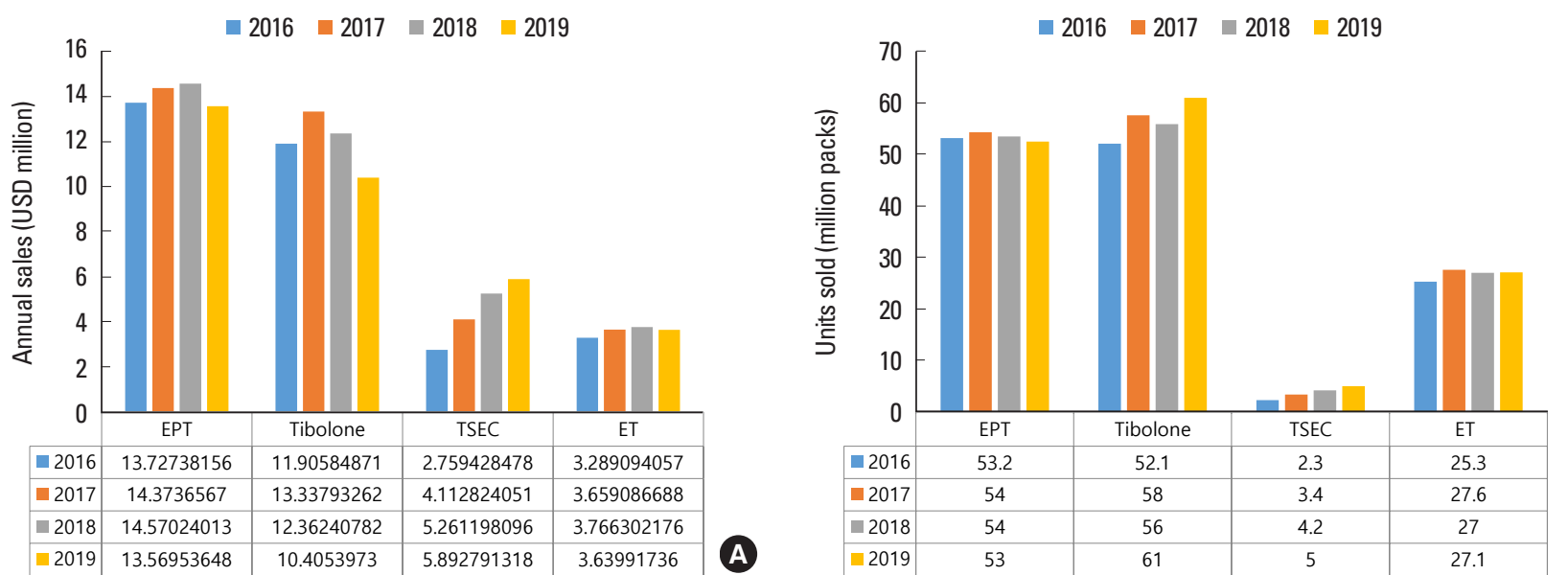

Fig. 1. The annual sales of postmenopausal hormones in USD (A) and units sold (B) from 2016 to 2019. EPT, estrogen-progestogen therapy; TSEC, tissue-selective estrogen complex; ET, estrogen therapy. 


\section{JBM}

2.3 million packs (USD 2.76 million) in 2016 to 5.0 million packs (USD 5.89 million) in 2019. The sales of EPT and ET are consistent from 2016 to 2019. In the case of tibolone, its sales declined in 2018 but increased again in 2019.

\section{Osteoporosis medications}

The Korean osteoporosis medication market, excluding postmenopausal hormones, was valued at almost USD 133.4 million in 2016 and increased reaching USD 200.1 million in 2019.

Excluding the postmenopausal hormone, bisphosphonates were the most widely sold medications for the treatment of osteoporosis, which sold 186.13 million units (USD 102.46 million) in 2016 and increased to 218.98 million units (USD 115.79 million) in 2019. SERM was the second most purchased medication with 30.31 million units sold (USD 18.82 million) in 2016 and increased to 42.52 million units sold (USD 22.76 million) in 2019. The sales of teriparatide also increased, and, notably, denosumab sales have increased 235 times from 150,000 units (USD 0.23 million) in 2016 to 49.4 million units (USD 40.57 million) in 2019 (Fig. 2).

Among the bisphosphonate medications, ibandronate and zoledronate have increased annually, while alendronate and risedronate have decreased. In 2016, alendronate sales amounted to 49.74 million units (USD 30.15 million); however, the amount decreased to 44.56 million units (USD 26.83 million) in 2019 (Fig. 3).

The increased sales of ibandronate were more pronounced with injectable preparation than oral preparations. Oral
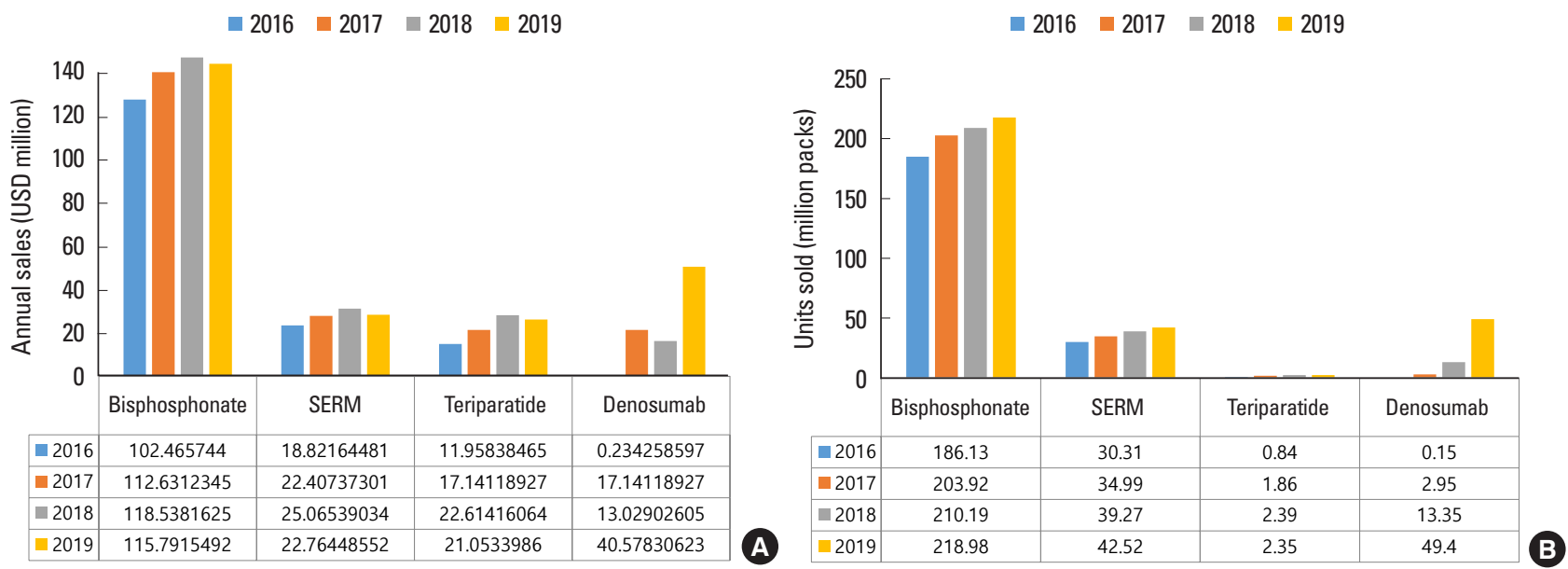

Fig. 2. The annual sales of osteoporosis medications in USD (A) and units sold (B) from 2016 to 2019. SERM, selective estrogen receptor modulator.
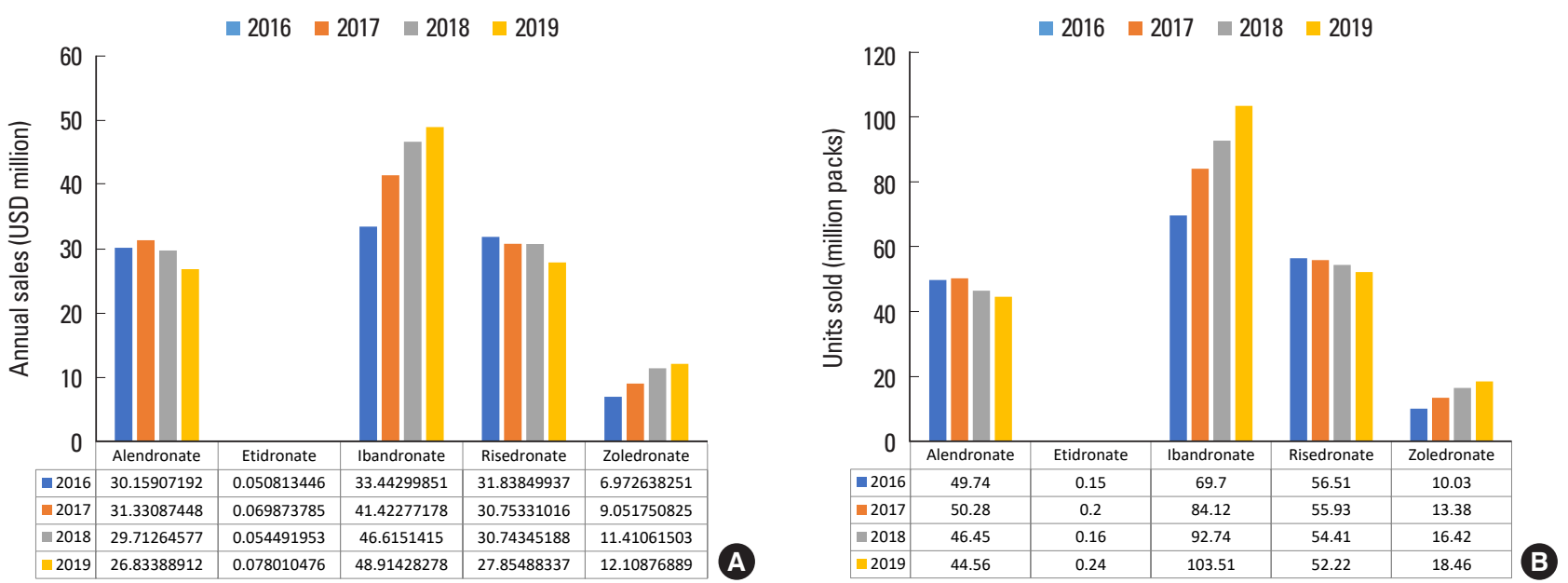

Fig. 3. The annual sales of bisphosphonates in USD (A) and units sold (B) from 2016 to 2019 

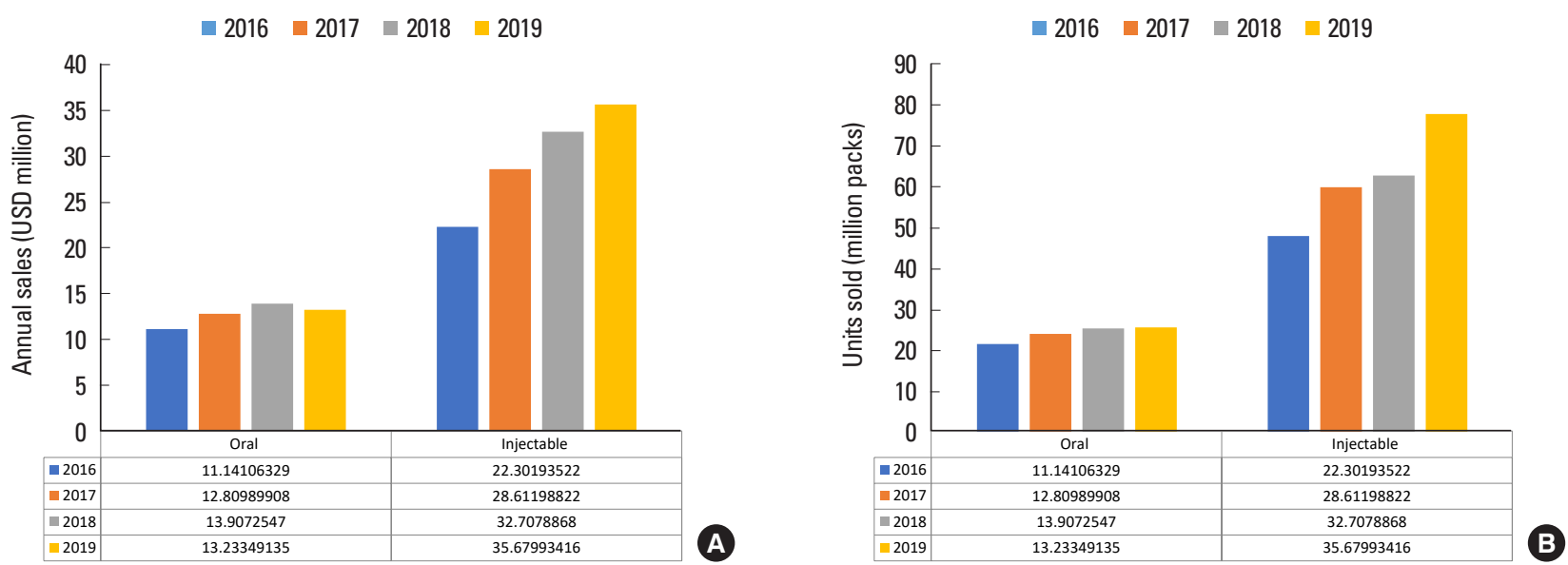

Fig. 4. The annual sales of ibandronate in USD (A) and units sold (B) from 2016 to 2019.

ibandronate sales amounted to 21.70 million units (USD 11.14 million) in 2016 and increased to 25.71 million units (USD 13.23 million) in 2019. Meanwhile, injectable ibandronate sales amounted to 48.0 million units (USD 22.30 million) in 2016 and increased to 77.79 million units (USD 35.67 million) in 2019 (Fig. 4).

\section{DISCUSSION}

There have been several reports on the use of osteoporosis medication in Korea.[7-9] Park [7] reported osteoporosis treatments with hormone therapy between 2004 and 2008. In the report, the most widely used osteoporosis medication was bisphosphonate. Regarding postmenopausal hormone therapy, EPT and ET have steadily decreased, while the use of tibolone increased steadily since the World Health Organization reported adverse effects from hormone therapy. In 2020, Lee et al. [9] reported the osteoporosis medication trends between 2006 and 2018, including bisphosphonates, calcitonin, denosumab, PTH, and SERMs, excluding menopausal hormones. Bisphosphonates were still the most widely sold medication in that report, although the percentage of bisphosphonates in all osteoporosis medications decreased since 2012.

This study's results included menopausal hormones, including TSEC, because postmenopausal hormones were also used to prevent and treat osteoporosis in South Korea. Although menopausal hormones were generally prescribed to treat menopausal symptoms, such as vasomotor symptoms and vaginal atrophy, the possibility that they were also prescribed for the prevention or treatment of osteoporosis should be considered, making it reasonable for the menopausal hormone to be included in the data analysis of osteoporosis medication sales.

Moreover, results showed that TSEC sales had increased annually, while EPT sales had decreased steadily. TSEC, which consisted of conjugated estrogen with bazedoxifene, balances endometrial protection and therapeutic effects on vasomotor symptoms and bone. Using TSEC is, at least, as efficacious in inhibiting the proliferation of breast cancer cells as raloxifene and does not increase breast density, breast pain or tenderness, or the risk of breast cancer.[1012] TSEC's protective effect against breast cancer may have attributed to its steady sales increase in Korea. According to Chae et al. [13], the most common reason for postmenopausal Korean women's unwillingness to undergo hormone therapy was their concern about the therapy's adverse effects and their indefinite fear of cancer. Thus, TSEC's sales are expected to increase because of its lower cancer risk and endometrium protection.

Meanwhile, bisphosphonates were still the most widely purchased among other osteoporosis medications. Although overall bisphosphonate sales have steadily increased, alendronate and risedronate sales decreased, while injectable ibandronate and zoledronate sales have increased. Poor compliance is a critical limiting factor in osteoporosis treatment. Thus, bisphosphonates with longer administration intervals have been introduced and showed higher compliance than with oral bisphosphonates.[14-16] Lee et al. [17] reported that regardless of bisphosphonate type, com- 
pliance plays a vital role in improving osteoporotic patients' health-related quality of life. Compliance is the main issue in the prescription of bisphosphonate, and the result of this study regarding the sales market reflected the problem of compliance.

Denosumab is a human monoclonal antibody targeting the key bone resorption mediator, RANKL. The drug is administered via subcutaneous injection every six months and is approved for treating various indications, including the treatment of postmenopausal women with osteoporosis. One study reported that denosumab is more effective in improving BMD than bisphosphonate, especially in women who switched from a bisphosphonate regimen, and that denosumab is well tolerated over up to 10 years of treatment.[18] Hadji et al. [19] described that the 2-year persistence of denosumab was 1.5 to 2 times higher than that of bisphosphonate, and the risk of discontinuation was significantly lower for denosumab than for bisphosphonates.

Previously in Korea, denosumab had limited osteoporosis treatment indications and was only approved for insurance for cases, such as the treatment failure with bisphosphonates or the contraindications of bisphosphonates, until March 2019.[20] Since April 2019, it has been covered by national insurance for every osteoporosis and was included in the medication options for osteoporosis. These factors, such as effectiveness, compliance, and insurance coverage, may have resulted in a marked increase in the use of denosumab.

The research is limited by 2 factors. First, the research did not include calcitonin, although it is still prescribed to some patients rarely; thus, this study does not reflect the whole osteoporosis medication market. Second, this study did not analyze the trend for annual percent change because this data has only 4 points from 2016 to 2019, which is not enough to analyze the trend.

In addition, TSEC formulation has been out of stock since May 2020, and denosumab began to apply for health insurance benefits from April 2019.

Future studies need to consider these issues the COVID-19 pandemic outbreak and a new sclerostin antibody drug called romosozumab, introduced to Korea in 2020. Moreover, by comparing the number of osteoporosis patients with the number of patients with osteoporosis, further studies are needed to determine whether the increase in sales prices is due to an increased number of patients or inflation.

In conclusion, the sales of osteoporosis medications have increased steadily. Moreover, the sales of TSEC, injectable bisphosphonates, and denosumab have increased annualIy. In contrast, the sales of EPT, ET, oral bisphosphonate have not increased as reflected on the trends of hormone therapy and osteoporosis medication markets.

\section{DECLARATIONS}

\section{Funding}

The authors received no financial support for this article.

\section{Ethics approval and consent to participate}

This study was granted exemption by Soonchunhyang University Seoul Hospital Institutional Review Board (IRB no. 2021-02-017).

\section{Conflict of interest}

No potential conflict of interest relevant to this article was reported.

\section{ORCID}

Eun Sil Lee https://orcid.org/0000-0003-4132-5533 Hyoung Moo Park https://orcid.org/0000-0003-0767-7711

\section{REFERENCES}

1. Ha YC. Epidemiology of osteoporosis in Korea. J Korean Med Assoc 2016;59:836-41. https://doi.org/10.5124/jkma. 2016.59.11.836.

2. Choi HJ, Shin CS, Ha YC, et al. Burden of osteoporosis in adults in Korea: a national health insurance database study. J Bone Miner Metab 2012;30:54-8. https://doi.org/10.1007/ s00774-011-0280-x.

3. Shin CS, Choi HJ, Kim MJ, et al. Prevalence and risk factors of osteoporosis in Korea: a community-based cohort study with lumbar spine and hip bone mineral density. Bone 2010;47:378-87. https://doi.org/10.1016/j.bone.2010.03.017.

4. Hinton PS, Nigh P, Thyfault J. Effectiveness of resistance training or jumping-exercise to increase bone mineral density in men with low bone mass: A 12-month randomized, clinical trial. Bone 2015;79:203-12. https://doi.org/10.1016/ j.bone.2015.06.008. 
5. Korean Society for Bone and Mineral Research. Physician's guide for osteoporosis. Seoul: Korean Society for Bone and Mineral Research; 2020.

6. Black DM, Rosen CJ. Clinical practice. Postmenopausal osteoporosis. N Engl J Med 2016;374:254-62. https://doi.org/ 10.1056/NEJMcp1513724.

7. Park HM. Current use of drugs for osteoporosis in Korea. Korean J Obstet Gynecol 2010;53:152-9. https://doi.org/ 10.5468/kjog.2010.53.2.152.

8. Park HM, Lee ES, Kim SM. The use of osteoporosis medications in Korea in 2008. Korean J Bone Metab 2009;16:8793.

9. Lee N, Choi YJ, Chung YS. The secular trends in the use of medications for osteoporosis in South Korea using Intercontinental Medical Statistics Health Sales Audit 20062018. Osteoporos Sarcopenia 2020;6:185-90. https://doi. org/10.1016/j.afos.2020.11.007.

10. Berrodin TJ, Chang KC, Komm BS, et al. Differential biochemical and cellular actions of Premarin estrogens: distinct pharmacology of bazedoxifene-conjugated estrogens combination. Mol Endocrinol 2009;23:74-85. https:// doi.org/10.1210/me.2008-0366.

11. Pickar JH, Boucher M, Morgenstern D. Tissue selective estrogen complex (TSEC): a review. Menopause 2018;25:103345. https://doi.org/10.1097/gme.0000000000001095.

12. Lee DY. Tissue-selective estrogen complex and breast. J Menopausal Med 2020;26:99-103. https://doi.org/10.6118/ jmm.20015.

13. Chae HD, Choi SY, Cho EJ, et al. Awareness and experience of menopausal symptom and hormone therapy in Korean postmenopausal women. J Menopausal Med 2014;20:713. https://doi.org/10.6118/jmm.2014.20.1.7.

14. Lee YK, Nho JH, Ha YC, et al. Persistence with intravenous zoledronate in elderly patients with osteoporosis. Osteoporos Int 2012;23:2329-33. https://doi.org/10.1007/s00198011-1881-x.

15. Cramer JA, Amonkar MM, Hebborn A, et al. Compliance and persistence with bisphosphonate dosing regimens among women with postmenopausal osteoporosis. Curr Med Res Opin 2005;21:1453-60. https://doi.org/10.1185/ $030079905 \times 61875$.

16. Cooper A, Drake J, Brankin E. Treatment persistence with once-monthly ibandronate and patient support vs. onceweekly alendronate: results from the PERSIST study. Int J Clin Pract 2006;60:896-905. https://doi.org/10.1111/j.17421241.2006.01059.x.

17. Lee YJ, Park CH, Lee YK, et al. Which bisphosphonate? It's the compliance!: Decision analysis. J Bone Metab 2016;23: 79-83. https://doi.org/10.11005/jbm.2016.23.2.79.

18. Deeks ED. Denosumab: A review in postmenopausal osteoporosis. Drugs Aging 2018;35:163-73. https://doi.org/ 10.1007/s40266-018-0525-7.

19. Hadji P, Kyvernitakis I, Kann PH, et al. GRAND-4: the German retrospective analysis of long-term persistence in women with osteoporosis treated with bisphosphonates or denosumab. Osteoporos Int 2016;27:2967-78. https:// doi.org/10.1007/s00198-016-3623-6.

20. Korean Society for Bone and Mineral Research. Physician's guide for osteoporosis. Seoul: Korean Society for Bone and Mineral Research; 2018. 